The biodegradable self-reinforced poly-DL-lactic acid spiral stent compared with a suprapubic catheter in the treatment of post-operative urinary retention after visual laser ablation of the prostate

\title{
Petas, Anssi
}

1997

Petas , A, Talja , M , Tammela , T LJ , Taari , K, Välimaa , T \& Törmälä , P 1997 , ' The biodegradable self-reinforced poly-DL-lactic acid spiral stent compared with a suprapubic catheter in the treatment of post-operative urinary retention after visual laser ablation of the prostate ' , British journal of urology , vol. 80 , pp. 439-443 . https://doi.org/10.1046/j.1464-410X.1997.00308.x

http://hdl.handle.net/10138/253852

https://doi.org/10.1046/j.1464-410X.1997.00308.x

publishedVersion

Downloaded from Helda, University of Helsinki institutional repository.

This is an electronic reprint of the original article.

This reprint may differ from the original in pagination and typographic detail.

Please cite the original version. 


\title{
The biodegradable self-reinforced poly-DL-lactic acid spiral stent compared with a suprapubic catheter in the treatment of post-operative urinary retention after visual laser ablation of the prostate
}

\author{
A. PÉTAS, M. TALJA*, T.L.J. TAMMELA $\dagger$, K. TAARI, T. VÄLIMAA $\ddagger$ and P. TÖRMÄLÄ \\ Department of Urology, Helsinki University Central Hospital, Helsinki, *Department of Surgery, Urological Unit, Päijät-Häme Central \\ Hospital, Lahti, Finland, †Division of Urology, Tampere University Hospital, Department of Clinical Medicine, University of Tampere, \\ Tampere, Finland and $¥$ Institute of Biomaterials, Tampere University of Technology, Tampere, Finland
}

Objective To evaluate the efficacy and safety of a biodegradable self-reinforced poly-DL-lactic acid (SR-PLA) spiral stent compared with a suprapubic catheter after visual laser ablation of the prostate (VLAP) in a randomized study.

Patients and methods The study comprised 45 patients (mean age 67.6 years, range 52-81) with bladder outlet obstruction caused by benign prostatic hyperplasia who received either a SR-PLA stent (22 patients) and a suprapubic catheter, or a suprapubic catheter only (23 patients) after undergoing VLAP. The suprapubic catheter was removed when voiding started. The response to VLAP and the effect of the stent or catheter were assessed using symptom scores, peak flow rate $\left(Q_{\max }\right)$, post-void residual volume (PVR), stent degradation, infection and outcome.

Results Voiding started on the first or second day after treatment in 18 (median one day) of 22 patients with a spiral stent and in eight (median 6 days) of 23 patients with only the suprapubic catheter. The mean degradation time of the SR-PLA stent was 6 months; spiral fragments were found in the prostatic urethra or the urinary bladder in 20 of the patients on cystoscopy. Improvements in patient-weighted symptom score, mean $Q_{\max }$ and PVR were significant in both groups at the 6 month follow-up. The infection rate increased with the duration of suprapubic catheterization, from $25 \%$ ( $0-3$ days) to $44 \%$ ( $>3$ days). Stones were seen in two stented patients during the follow-up.

Conclusion The SR-PLA spiral stent is safe and effective for the treatment of post-operative urinary retention after laser therapy. The degradation time of the stent was unnecessarily long compared with the duration of prostatic swelling and tissue sloughing after laser therapy. The infection rate depends on the duration of suprapubic catheterization.

Keywords Biodegradable materials, laser therapy, prostate, prostatic hyperplasia, stents

\section{Introduction}

Visual laser ablation of the prostate (VLAP) has become an alternative for the treatment of bladder outlet obstruction caused by BPH. The first experimental studies in a canine model showed the potential of laser-induced prostatectomy [1] and the side-firing laser was first introduced into clinical use in Australia [2]. Postoperative catheterization is required for 4.7-7 days using a free-beam side-firing laser fibre [3,4], managed conventionally using an indwelling or suprapubic catheter. In 1980, Fabian introduced a urological spiral stent to keep the prostatic lobes from compressing the urethra, thus allowing spontaneous voiding [5]. Several other investi-

Accepted for publication 15 April 1997

CC 1997 British Journal of Urology gators have reported good results with similar spiral stents for transient or definitive therapy of prostatic obstruction [6,7]. A biodegradable spiral stent made from self-reinforced polyglycolic acid (SR-PGA) was reported to be effective in the prevention of the postoperative urinary retention after VLAP by Talja et al. [8]. The degradation time of the SR-PGA spiral stent is 2-4 weeks and longer than the mean duration of catheterization after VLAP. As the degradation time of the SR-PGA stent was considered too short, a new compound with a longer degradation time was selected. The degradation time can be changed by selecting an appropriate bioabsorbable material from which to manufacture the stents. A spiral stent made from poly-DLlactic acid has a degradation time of more than 3 months in vitro. A previous experimental study has shown that 
self-reinforced poly-L-lactic acid (SR-PLA) is biocompatible in the anterior urethra [9].

Thus, the aim of the present study was to evaluate the efficacy and safety of the SR-PLA spiral stent compared with the suprapubic catheter in the prevention of urinary retention after VLAP.

\section{Patients and methods}

\section{Materials}

Poly-DL-lactide is a copolymer of two stereoisomers of lactic acid; in the current stent the ratio of the $\mathrm{L}$ and $\mathrm{D}$ stereoisomers is 96:4. The spiral stents are manufactured by extrusion and die-drawing with a wire diameter of $1.1 \mathrm{~mm}$ and a draw ratio of 7 [10]. The configuration of the spiral resembled that of the stent described by Fabian [5]. The spiral was $8 \mathrm{~mm}$ in outer diameter and the prostatic portion was $45 \mathrm{~mm}$ long (Fig. 1). The spirals were sterilized by gamma irradiation. The spiral tends to straighten but maintain its configuration so that the outer diameter increased by about half during incubation for 14 days in phosphate-buffer solution $(\mathrm{pH} 6.1)$ at $37^{\circ} \mathrm{C}$ in vitro. The material is biocompatible and in vitro it degrades into lactic acid, mainly by hydrolysis [11]. Some enzymes in vivo, as well as elevated temperature in vitro, accelerate the degradation, the end-products of which are water and carbon dioxide [12]. The mechanical properties and the degradation time depend on the degree of polymerization, the internal arrangement of the material components, the site of implantation and the shape of the implant.

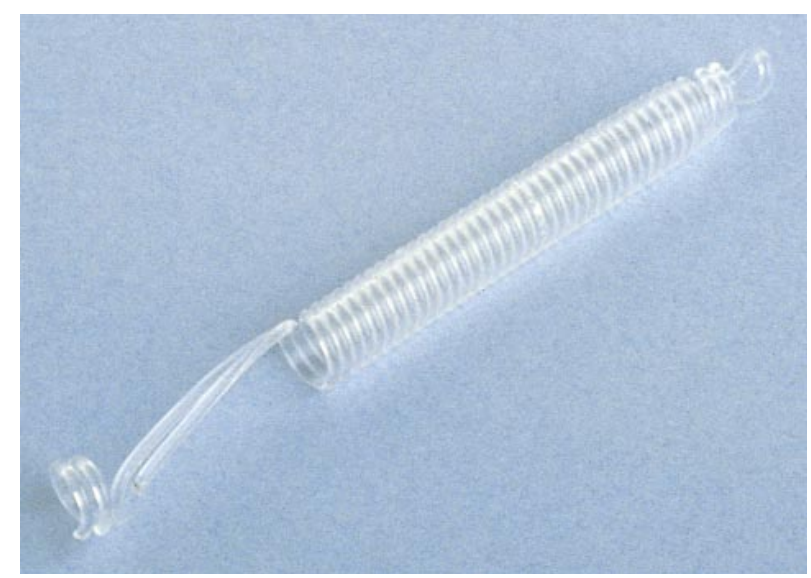

Fig. 1. The self-reinforced poly-DL-lactic (SR-PLA) spiral stent. The spiral is $8 \mathrm{~mm}$ in diameter and the prostatic part is $45 \mathrm{~mm}$ long. The diameter of the original wire is $1.1 \mathrm{~mm}$.

\section{Patients}

The study comprised 45 patients (mean age 67.6 years, range 52-81) with bladder outlet obstruction caused by $\mathrm{BPH} ; 22$ were randomized to receive a spiral stent and a suprapubic catheter, and 23 a suprapubic catheter only, after VLAP. The mean (range) prostatic weight was 35 (12-60) $\mathrm{g}$ in the stented patients and 40 (17-88) $\mathrm{g}$ in those with a suprapubic catheter only (no significant difference). One patient in the stent group and two patients in the suprapubic catheter group had urinary retention pre-operatively. Patients with malignancy of urinary bladder or prostate, chronic bacterial prostatitis, urethral stricture or previous surgery of prostate, or who were undergoing therapy with cholinergic, anticholinergic drugs or finasteride, were excluded from the study. The peak urinary flow rate $\left(Q_{\max }\right)$ was $<15 \mathrm{~mL} / \mathrm{s}$ with a voided volume of $>150 \mathrm{~mL}$. TRUS-guided biopsies were obtained if the PSA level was $>4.0 \mathrm{ng} / \mathrm{mL}$ or if the DRE or TRUS revealed any abnormality.

\section{Procedure}

The bladder was filled with saline during cystoscopy and a $12 \mathrm{~F}$ suprapubic balloon catheter inserted. VLAP was then performed with a free-beam side-firing tip (SideFiber $1800^{\circledR}$, Ceramoptec $\mathrm{GmbH}$ or Urolase ${ }^{\circledR}$, CR Bard, Inc) cooled by continuous irrigation with $37^{\circ} \mathrm{C}$ saline. Standard neodymium:YAG generators were used to provide the photothermal energy at a power setting of 40-50 W in continuous-wave mode. The laser was applied for $90 \mathrm{~s}$ for the lateral lobes and $45 \mathrm{~s}$ for the median lobe. Laser energy was delivered to the lateral lobes in four quadrants circumferentially at the 2, 4, 8 and 10 o'clock positions, in two planes when the prostatic urethra was longer than $2.5 \mathrm{~cm}$. In addition, extra firing points were used to totally bleach the surface of the lateral lobes. After removing the laser fibre, a 3-5 F ureteric catheter was inserted through the cystoscope into the bladder and the cystoscope removed. Using the tip of the cystoscope, a SR-PLA spiral stent was inserted into the prostatic urethra along the ureteric catheter. The correct location of the stent was verified endoscopically. The patient was allowed to void immediately if possible and the suprapubic catheter was removed when the patient was able to void freely. All patients were given ciprofloxacillin $200 \mathrm{mg}$ intravenously or $500 \mathrm{mg}$ by mouth peri-operatively, except one patient in the spiral stent group who was given cefuroxim $1.5 \mathrm{~g}$. Two patients from each group had no antibiotic peri-operatively. The antibiotic treatment continued as prophylaxis, using various antibiotics, for 2 weeks in all patients. The criterion for urinary tract infection was defined as $10^{5}$ c.f.u. in culture. 
The follow-up assessment consisted of uroflowmetry, ultrasonographic estimation of post-void residual volume (PVR), the analysis of serum creatinine and c-reactive protein, urine culture, a patient-weighted symptom score questionnaire (DAN-PSS-1, including sexual scoring [13]) and cysto-urethroscopy. The symptom score was used as it includes a bother score in addition to the symptom score and is therefore considered to provide a more reliable estimate of symptoms than do other scoring systems [13]. The assessments were performed before and at 4 weeks, 3 and 6 months after VLAP; at 6 months, TRUS was also performed.

The paired $t$-test was used to evaluate differences between the assessments and the chi-square test used to evaluate differences in the start of voiding and the infection rates. A $P$ value $<0.05$ was considered to indicate statistically significant differences.

\section{Results}

All the patients completed the 6-month follow-up. The mean dose of the laser energy used was $1032 \mathrm{~J} / \mathrm{mL}$ (range 570-2670). Eighteen patients with a spiral stent voided freely on the first or second post-operative day (median 1 day, range 0-10), compared with eight of those with a suprapubic catheter (median 6 days, range $1-13 ; P<0.001)$. The suprapubic catheter was removed after a median of 2.5 days (range 1-29) in the spiral stent group and 12 days (range 2-51) in the suprapubic catheter group. Voiding was delayed in one patient in the spiral stent group for 5 days post-operatively, until a blood clot passed with the urine. No transfusions were required in any of the patients.

Endoscopically, the stents were intact and in the correct position at the 1 month follow-up; after 3 months the stents were partially degraded in all patients and fragments of the stent were still in the prostatic urethra or urinary bladder in 20 patients at the 6 month followup. Stones had formed in two patients in the spiral stent group at 6 months; the first patient had an asymptomatic stone in the urinary bladder, whereas the second patient had irritative symptoms from a stone in the prostatic urethra and five stones in the urinary bladder. These patients had no evidence of remnants of the spiral stent.

On fragmentation, the spiral stent initially tended to break in the straight part between the distal ring and prostatic part of the stent. The prostatic part then broke into large circular fragments (Fig. 2a) and finally, these circular fragments broke into smaller curved pieces 2-4 mm long (Fig. 2b). The stent fragments migrated into the urinary bladder or were voided with urine. Fragments were not removed during cystoscopy at the follow-up examinations.

In the spiral stent group, the change in $Q_{\max }$ was
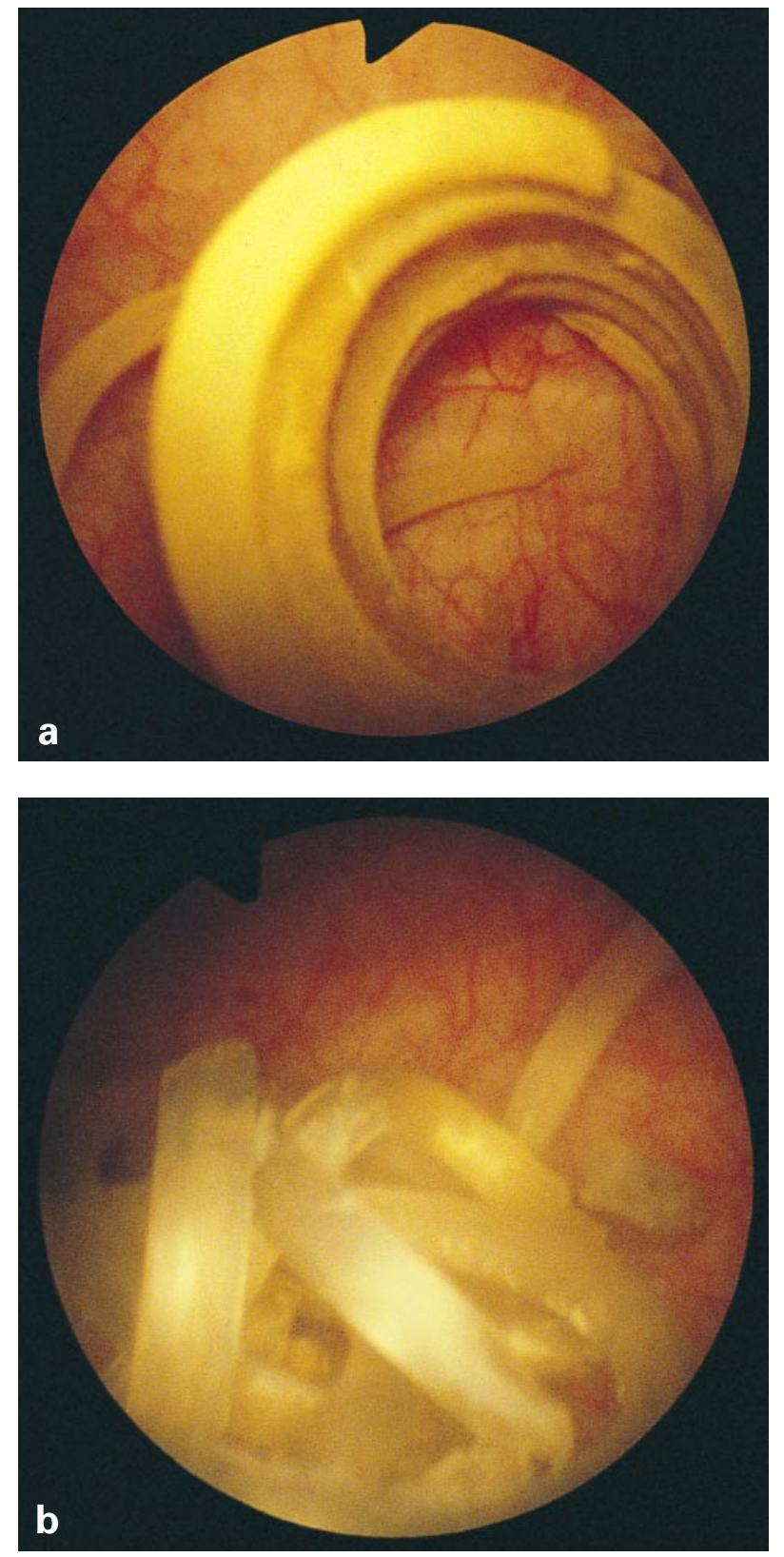

Fig. 2. a, Large circular fragments and b, small, curved fragments of the SR-PLA spiral stent in the urinary bladder at the 6-month follow-up.

highly significant at $1,3(P<0.001)$ and 6 months $(P<0.01)$, and in the suprapubic catheter group it was not significant at 1 month, but significant at 3 and 6 months (both $P<0.001$; Table 1). The mean PVR decreased from $132 \mathrm{~mL}$ to $45 \mathrm{~mL}(P<0.05)$ in the spiral stent group and from $139 \mathrm{~mL}$ to $40 \mathrm{~mL}(P<0.001)$ at 6 months. The patient-weighted prostatic symptom score decreased significantly at 6 months $(P<0.001)$ in both groups. There were no significant changes in the sexual symptom scores. There were no significant differences 
Follow-up (months)

\begin{tabular}{lllll}
\cline { 3 - 4 } Mean (range) & Pre-operative & 1 & 3 & 6 \\
\hline$Q_{\max }(\mathrm{mL} / \mathrm{s})$ & & & & \\
Spiral stent & $6.9(2.0-13.6)$ & $15.4(5.7-34.5) \dagger$ & $14.5(6.6-24.8) \dagger$ & $15.2(4.2-40.6) \dagger$ \\
Suprapubic & $7.5(3.2-14.7)$ & $11.1(2.2-48.8)$ & $14.6(5.6-26.7) \dagger$ & $16.4(4.9-38.0) \dagger$ \\
Symptom score & & & & \\
Spiral stent & $27.0(0-50)$ & $17.1(0-53)$ & $12.8(0-34)^{*}$ & $9.1(0-78) \dagger$ \\
Suprapubic & $22.1(4-55)$ & $13.3(0-47)$ & $10.6(0-28)$ & $4.4(0-16) \dagger$ \\
& & & &
\end{tabular}

${ }^{*} P<0.01, \dagger P<0.005$; comparison with the pre-operative value of the same treatment group. Differences between the groups at the various times were not statistically significant.
Table 1 Mean values and ranges of peak urinary flow rate $\left(Q_{\max }\right)$ and symptom score during follow-up between the groups for $Q_{\max }$, PVR or symptom scores during the follow-up.

A UTI was detected in nine of 22 patients in the spiral stent group and in eight of 23 in the suprapubic catheter group during the follow-up. For all patients, a UTI occurred in nine patients in more than two assessments and in five patients in only one assessment during the follow-up. When infections were categorized according to the duration of suprapubic catheterization, the infection rate was three of 20 in the those catheterized for 0-3 days and 14 of 25 in those catheterized for $>3$ days $(P=0.002)$.

\section{Discussion}

Although VLAP offers some advantages over TURP, it induces high temperatures in prostatic tissue, causing acute tissue swelling and producing urinary retention in most patients. It is conventionally managed using an indwelling or suprapubic catheter; the mean duration of catheterization is $4-7$ days $[3,4,14]$. Such prolonged indwelling or suprapubic catheterization after VLAP is inconvenient for the patient and the catheter provides a route for bacterial colonization of the urinary bladder. Infection of the necrotic tissue may even lead to prolonged urinary infection. An infection rate of 50\% without antibiotic prophylaxis has been reported [15]. The incidence of post-operative infection was higher in patients catheterized suprapubically for $>3$ days in the present study, suggesting that infection associated with VLAP arises by contamination from bacteria migrating through the suprapubic catheter. As the need for suprapubic catheterization varies with the patient, and the laser procedure is easier to perform with continuous irrigation, the suprapubic catheter could be inserted later in cases with urinary retention; the infection rate could thus be reduced.

Voiding did not begin immediately after VLAP in 10 of the 22 patients in the spiral stent group, but it was earlier than in the suprapubic catheter group. The spiral stent is so rigid that it cannot be closed by compression of the prostatic side lobes. The short length of the spiral stent may be the most important reason for delayed voiding. The spiral shortens when it dilates, by about $40 \%$ of its original length (the length of the undilated spiral was $45 \mathrm{~mm}$ ); currently, longer stents are recommended. It is also probable that sloughing tissue and occasional haemorrhage from prostatic lobes tend to obstruct the spiral stent, causing a delay in the start of voiding. None of the spiral stents migrated in the present study; the expansion of about $50 \%$ in diameter secures it in about 2 weeks. There was no need to remove the device in any patient; polymer fragments, if left in the bladder, degraded completely within 6-8 months. Encrustation and stone formation occur frequently on metallic stents [16,17], but the incidence of these was low in the present study; two patients formed stones where stent fragments were no longer present. Infection can induce urinary stone formation and it may accelerate degradation of the spiral stent by increasing the $\mathrm{pH}$ of the urine.

The laser technique used was similar to that in other VLAP studies, and with a comparable energy dose; the outcome of the present VLAP agrees with the results reported by others $[3,4]$. Overall, the decrease in voiding symptoms was highly significant.

The SR-PLA spiral stent could also be used to prevent urinary retention in combination with other therapies that might induce prostatic oedema, e.g. transurethral microwave therapy, transurethral needle ablation, highintensity focused ultrasound, interstitial laser coagulation of the prostate and cryotherapy. Other indications for the spiral stent might be recurrent bladder neck stricture, temporary treatment of urinary retention in patients awaiting surgery, or in the early-phase resolution of $\mathrm{BOO}$ in patients waiting for medical therapy to take effect.

In conclusion, the SR-PLA is safe and effective compared with the suprapubic catheter in the treatment of post-operative urinary retention after VLAP. The 
degradation time of the SR-PLA 96 spiral stent was longer than the duration of prostatic swelling and tissue sloughing after VLAP and longer than the time needed to prevent post-operative urinary retention; however, there was no need to remove the spiral stent. A spiral stent with a shorter degradation time, e.g. a selfreinforced polyglycolic acid (degradation time 2-4 weeks) or faster degrading SR-PLA spirals, may be better for the treatment of urinary retention after VLAP. Therefore, further studies are needed to modify the spiral stent and to assess the most suitable materials for particular demands. New properties, such as radioopacity, modifications in configuration, tailored degradation sequences, and stents with fast expansion times, should be examined. Spiral stents also offer the possibility of the temporary stenting of tubular organs in other areas of surgery.

\section{References}

1 Roth R, Aretz H. Transurethral ultrasound-guided laserinduced prostatectomy (TULIP procedure): a canine prostate feasibility study. J Urol 1991; 146: 1128-35

2 Costello AJ, Bowsher WG, Bolton DM, Brasils KG, Burt J. Laser ablation of the prostate in patients with benign prostatic hypertrophy. Br J Urol 1992; 69: 603-8

3 Kabalin JN, Gill HS, Bite G, Wolfe V. Comparative study of laser versus electrocautery prostatic resection: 18-month followup with complex urodynamic assessment. J Urol 1995; 153: 94-8

4 Norris JP, Norris DM, Lee RD, Rubenstein MA. Visual laser ablation of the prostate: clinical experience in 108 patients. J Urol 1993; 150: 1612-4

5 Fabian KM. Der Intraprostatische 'Partielle Katheter' (Urologische Spirale). Urologe A 1980; 19: 236-8

6 Bajoria S, Agarwal SA, White R, Zafar F, Williams G. Experience with the second generation Urolume prostatic stent. Br J Urol 1995; 75: 325-7

7 Ala-Opas M, Talja M, Tiitinen J, Hellström P, Heikkinen A, Nurmi M. Prostakath in urinary outflow obstruction. Ann Chir Gynaecol 1993; 206 suppl: 14-8

8 Talja M, Tammela T, Pétas A et al. Biodegradable self- reinforced polyglycolic acid spiral stent in prevention of postoperative urinary retention after visual laser ablation of the prostate-laser prostatectomy. J Urol 1995; 154: 2089-92

9 Kemppainen E, Talja M, Riihelä M, Pohjonen T, Törmälä P, Alfthan O. A bioresorbable urethral stent. Urol Res 1993; 21: $235-8$

10 Törmälä P. Biodegradable self-reinforced composite materials; manufacturing structure and mechanical properties. Clin Mater 1992; 10: 29-34

11 Chu CC. Hydrolytic degradation of polyglycolic acid: tensile strength and crystallinity study. J Appl Polym Sci 1981; 26: 1727-34

12 Chu CC, Williams DF. The effect of gamma irradiation on the enzymatic degradation of polyglycolic acid absorbable sutures. J Biomed Mater Res 1983; 17: 1029-40

13 Meyhoff HH, Hald T, Nordling J, Andersen JT, Bilde T, Walter S. A new patient weighted symptom score system (DAN-PSS-1). Clinical assessment of indications and outcomes of transurethral prostatectomy for uncomplicated benign prostatic hyperplasia. Scand J Urol Nephrol 1993; 27: 493-9

14 Kabalin J, Bite G, Doll S. Neodymium: YAG laser coagulation prostatectomy: 3 years experience with 227 patients. J Urol 1996; 155: 181-5

15 Costa FJ. Lomefloxacin prophylaxis in visual laser ablation of the prostate. Urology 1994; 44: 933-6

16 Holmes SAV, Miller PD, Crocker PR, Kirby RS. Encrustation of intraprostatic stents-a comparative study. $\mathrm{Br}$ J Urol 1992; 69: 383-7

17 Keane PF, Bonner MC, Johnston SR, Zafar A, Gorman SP. Characterisation of biofilm and encrustation on uretic stents in vivo. Br J Urol 1992; 73: 687-91

\section{Authors}

A. Pétas, MD, Consultant Urologist.

M. Talja, MD, PhD, Senior Lecturer.

T.L.J. Tammela, MD, PhD, Senior Lecturer.

K. Taari, MD, PhD, Consultant Urologist.

T. Välimaa, GE (Eng), Project Manager.

P. Törmälä, Professor.

Correspondence: Dr A. Pétas, Department of Urology, Helsinki University Central Hospital, Haartmaninkatu 4, FIN-00290 Helsinki, Finland. 